\title{
Viscoelastic Plate Analysis Based on Gâteaux Differential
}

\author{
Fethi Kadıoğlu and Gülçin Tekin \\ Istanbul Technical University, Civil Engineering Department, Istanbul, Turkey
}

\begin{abstract}
In this study, it is aimed to analyze the quasi-static response of viscoelastic Kirchhoff plates with mixed finite element formulation based on the Gâteaux differential. Although the static response of elastic plate, beam and shell structures is a widely studied topic, there are few studies that exist in the literature pertaining to the analysis of the viscoelastic structural elements especially with complex geometries, loading conditions and constitutive relations. The developed mixed finite element model in transformed Laplace-Carson space has four unknowns as displacement, bending and twisting moments in addition to the dynamic and geometric boundary condition terms. Four-parameter solid model is employed for modelling the viscoelastic behaviour. For transformation of the solutions obtained in the Laplace-Carson domain to the time domain, different numerical inverse transform techniques are employed. The developed solution technique is applied to several quasi-static example problems for the verification of the suggested numerical procedure.
\end{abstract}

\section{Introduction}

Determination of the behaviour of plate elements is an important engineering problem due to their wide application in all fields of engineering. To simplify the analysis, it is generally assumed that the material is elastic. However, most engineering materials are viscoelastic due to internal friction. Therefore, viscoelastic constitutive relation is more realistic than the elastic constitutive relation when considering the material behaviour. There are many works in the literature on the theory of viscoelasticity [1-2].

For the problems which have complex geometries and constitutive relations, closed form solutions are often not possible and numerical solution methods should be employed. The application of the numerical methods to viscoelastic problems has been presented by a number of authors [3-4]. However, to the best of the present authors' knowledge, there are very few published studies on the viscoelastic analysis of plates [5-9].

In this study, quasi-static response of viscoelastic plates under time dependent loads are investigated in the Laplace-Carson domain based on the Gâteaux differential method. The Gâteaux differential method is a more powerful, reliable and efficient variational tool when formulating the mixed type finite elements. Based on the Gâteaux differential method, Aköz and his co-workers [913] analysed the quasi- static and dynamic behaviour of the viscoelastic beam and plate elements by employing the Kelvin and/or Three-parameter Kelvin model. The aim of the present work is to demonstrate the application of an efficient method, as mentioned above, to the viscoelastic Kirchhoff plates with the model of fourparameter solid type. The solutions obtained in the
Laplace-Carson space are transformed to the real space using different (MDOP, Dubner and Abate and Durbin) numerical inverse Laplace transform methods. Numerical results for quasi-static analysis of viscoelastic plates are presented.

\section{Governing Equations}

Considering a plate with the thickness $\mathrm{h}$ and the positive directions of internal forces are illustrated in Figure 1. According to the classical thin plate theory (Kirchhoff's plate theory) the influence of the transverse shear deformation on the deflection of the plate can be neglected.

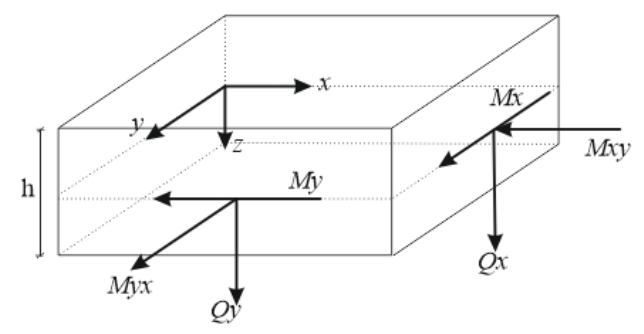

Figure 1. Initial Forces.

The equilibrium equation of the elastic thin plate can be given as follows [14-15]:

$$
\frac{\partial^{2} M_{x}}{\partial x^{2}}+\frac{\partial^{2} M_{y}}{\partial y^{2}}+2 \frac{\partial^{2} M_{x y}}{\partial x \partial y}+q=0
$$

where $q$ indicates the normal load distribution and $M_{x}, M_{y}$, $M_{x y}$ are internal moments. Adopting the Kirchhoff 
hypothesis, the bending and torsion moments of viscoelastic plates can be written with two operators $E_{1}^{*}$ and $E_{2}^{*}$ in hereditary integral form $[7,9]$ as follows:

$$
\begin{aligned}
& M_{x}=-\frac{h^{3}}{12}\left[E_{1}^{*} \frac{\partial^{2} w}{\partial x^{2}}+E_{2}^{*} \frac{\partial^{2} w}{\partial y^{2}}\right] \\
& M_{y}=-\frac{h^{3}}{12}\left[E_{1}^{*} \frac{\partial^{2} w}{\partial y^{2}}+E_{2}^{*} \frac{\partial^{2} w}{\partial x^{2}}\right] \\
& M_{x y}=-\frac{h^{3}}{12}\left[E_{1}^{*}-E_{2}^{*}\right] \frac{\partial^{2} w}{\partial x \partial y}
\end{aligned}
$$

where $\mathrm{w}$ is the vertical displacement function of the plate' middle surface. Operating the Laplace-Carson transform in order to remove the time derivatives from governing equations, Equations (1) and (2) yields:

$$
\begin{aligned}
& -\frac{\partial^{2} \bar{M}_{x}}{\partial x^{2}}-\frac{\partial^{2} \bar{M}_{y}}{\partial y^{2}}-2 \frac{\partial^{2} \bar{M}_{x y}}{\partial x \partial y}=\bar{q} \\
& -\bar{M}_{x}-\bar{D}^{\star}\left(\frac{\partial^{2} \bar{w}}{\partial x^{2}}+v \frac{\partial^{2} \bar{w}}{\partial y^{2}}\right)=0 \\
& -\bar{M}_{y}-\bar{D}^{\star}\left(\frac{\partial^{2} \bar{w}}{\partial y^{2}}+v \frac{\partial^{2} \bar{w}}{\partial x^{2}}\right)=0 \\
& -\bar{M}_{x y}-(1-v) \bar{D}^{\star} \frac{\partial^{2} \bar{w}}{\partial x \partial y}=0
\end{aligned}
$$

where $\bar{D}^{*}$ is related with the creep function $\bar{Y}^{\star}$ as $\bar{D}^{*}=\frac{h^{3}}{12} \bar{Y}^{*}$.The dynamic $\quad \bar{T}=\hat{\bar{T}},-\bar{M}=-\hat{\bar{M}} \quad$ and geometric $\quad \bar{W}^{\prime}=\hat{\bar{W}}^{\prime},-\bar{W}=-\hat{\bar{W}}$ boundary conditions are defined in the Laplace-Carson space to complete the field equations.

Field equations can be written in operator form as:

$$
\bar{Q}=\bar{L} \bar{u}-\bar{f}
$$

where $\overline{\mathbf{Q}}$ is a potential if the equality [16]:

$$
<d \overline{\mathbf{Q}}\left(\overline{\boldsymbol{u}}, \overline{\boldsymbol{u}}^{\prime}\right), \overline{\boldsymbol{u}}^{*}>=<d \overline{\mathbf{Q}}\left(\overline{\boldsymbol{u}}, \bar{u}^{*}\right), \overline{\boldsymbol{u}}^{\prime}>
$$

is satisfied. Where $\boldsymbol{d} \overline{\boldsymbol{Q}}\left(\overline{\boldsymbol{u}}, \overline{\boldsymbol{u}}^{\prime}\right)$ is the Gâteaux derivative of $\overline{\boldsymbol{Q}}$ and parenthesis indicate the inner products. After satisfying the requirement, the functional corresponding to the field equations is obtained as:

$$
I(\overline{\boldsymbol{u}})=\int_{0}^{1}<\overline{\boldsymbol{Q}}(\psi \overline{\boldsymbol{u}}, \overline{\boldsymbol{f}}), \overline{\boldsymbol{u}}>d \psi
$$

where $\psi$ is a scalar quantity. The explicit form of the functional corresponding to the field equations of the viscoelastic Kirchhoff plates in the Laplace-Carson space is obtained as:

$$
\begin{aligned}
& I(\bar{u})=\left[\bar{w}_{, x}, \bar{M}_{x_{x}}\right]+\left[\bar{w}_{, y}, \bar{M}_{y_{y}}\right]+\left[\bar{w}_{, x}, \bar{M}_{x y_{y}}\right] \\
& +\left[\bar{w}_{, y}, \bar{M}_{x y_{x}}\right]-[\bar{q}, \bar{w}]-\frac{1}{2 \bar{D}\left(1-v^{2}\right)}\left\{\left[\bar{M}_{x}, \bar{M}_{x}\right]+\left[\bar{M}_{y}, \bar{M}_{y}\right]\right\} \\
& +\frac{v}{\bar{D}\left(1-v^{2}\right)}\left[\bar{M}_{x}, \bar{M}_{y}\right]-\frac{1}{\bar{D}(1-v)}\left[\bar{M}_{x y}, \bar{M}_{x y}\right] \\
& -[\hat{\bar{T}}, \bar{W}]_{\sigma}-\left[(\bar{M}-\hat{\bar{M}}), \bar{W}^{\prime}\right]_{\sigma}-\left[\hat{\bar{W}}^{\prime}, \bar{M}\right]_{\varepsilon}-[(\bar{W}-\hat{\bar{W}}), \bar{T}]_{\varepsilon}
\end{aligned}
$$

where subscript $\sigma$ represents dynamic boundary condition, $\varepsilon$ represents geometric boundary condition.

The functional variables in Eq. (7) are in the LaplaceCarson space. The four variables of the functional are expressed by the shape functions which are defined for the rectangular master element (Figure 2) as below:

$$
\begin{aligned}
& N_{1}=(1-\xi)(1-\eta) \\
& N_{2}=\xi(1-\eta) \\
& N_{3}=(1-\xi) \eta \\
& N_{4}=\xi \eta
\end{aligned}
$$

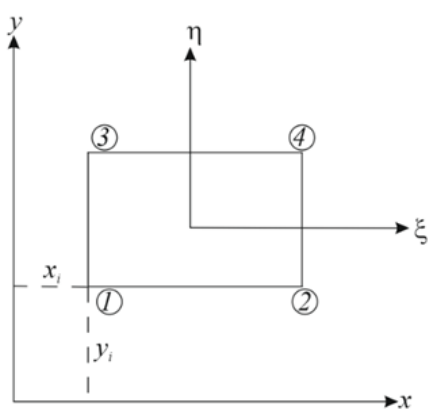

Figure 2. Rectangular Master Element

All expressions of unknown and known quantities in terms of shape functions are inserted into Eq. (7) and simplifying with respect to nodal variables; the element matrix is derived. The numerical solutions of the finite element formulation derived in Laplace-Carson space are transformed to the real time domain using the inverse numerical Laplace transformation. In this study, Maximum Degree of Precision (MDOP), Durbin's, and Dubner and Abate's methods are employed. Papers for discussion of Laplace inversion process, see [17-20].

\section{Numerical Example}

The performance of the proposed method is tested through various problems. In all applications, Fourparameter Kelvin (FPK) model as illustrated in Figure 3 is employed.

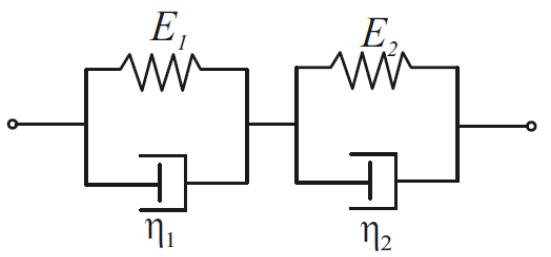

Figure 3. Four-Parameter Solid Model.

The material coefficients are chosen as follows:

- The Four-Parameter solid model:

$\mathrm{E}_{1}=98 \mathrm{MPa}, \eta_{1}=98 \mathrm{MPa} . \mathrm{s}, \mathrm{E}_{2}=274.4 \mathrm{MPa}, \eta_{2}=274.4$ MPa.s, $v=0.3$

The relaxation modulus of Four-parameter Kelvin material is as follows:

$$
J_{(t)}=\frac{1}{E_{1}}\left(1-e^{-\frac{E_{1}}{\eta_{1}} t}\right)+\frac{1}{E_{2}}\left(1-e^{-\frac{E_{2}}{\eta_{2}} t}\right)
$$


In all applications, due to the symmetry the quarter of simply supported rectangular plate with length $\mathrm{a}=4 \mathrm{~m}$, width $\mathrm{b}=4 \mathrm{~m}$ and thickness $\mathrm{h}=0.1 \mathrm{~m}$ is solved for different time-dependent loads by using equal rectangular mesh sizes $(4 \times 4$ mesh scheme). Recently, a numerical example for determination of the most suitable mesh scheme was presented by [9]. According to the results of that study, the $4 \times 4$ mesh scheme results are very satisfactory and this scheme has the advantage of saving time. Throughout this study, all numerical examples are solved for $\mathrm{aT}=5, \mathrm{~T}=20$ and $\mathrm{N}=100$ to decrease the fluctuation in the solutions. For more information about the most suitable values for the effective parameters of the inverse transform techniques, see [9].

In all numerical examples, the displacement value is given for the middle point of the plate by taking the advantage of symmetry property. The time histories of loads in applications are sketched in Figure 4.
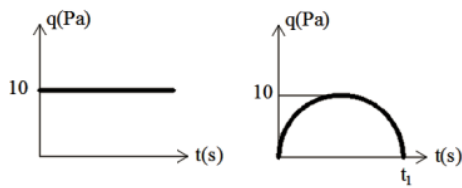

Type I
(Step Load)

$$
\text { Type II }
$$

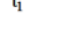

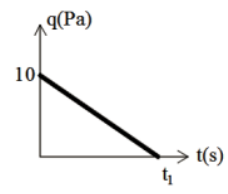
Type III
(Triangular Impulsive Load)
Figure 4. Time histories of external loads.

\section{Example 1}

A simply supported viscoelastic rectangular plate subjected to step load (Type I) is considered. The central displacement obtained with the MDOP, Dubner \& Abate's and Durbin's inverse transform techniques is shown in Figure 5 for the ratio between the viscosity coefficient $(\eta)$ and modulus of elasticity of the material (E) equals to $0.1 \quad\left(\eta_{1} / \mathrm{E}_{1}=\eta_{2} / \mathrm{E}_{2}=0.1\right)$. This example is solved in order to test the success of the inverse Laplace transform methods. Results show that all considered transform methods give good results for the time dependent displacement at the center of the plate for the step load.

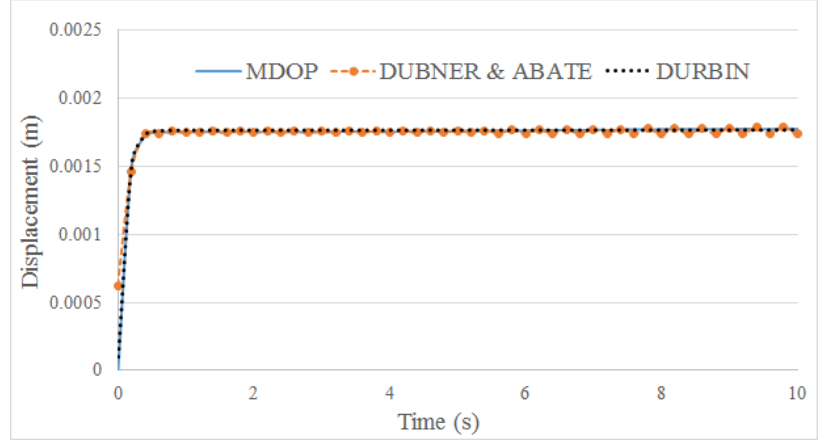

Figure 5. Comparison of different inverse Laplace transform techniques for the load Type I.

\section{Example 2}

A simply supported viscoelastic rectangular plate under the sinusoidal impulsive load (Type II) for $t_{1}=10 \mathrm{~s}$ is considered. The time-dependent displacement and bending moment at the center of the plate are obtained with the MDOP, Dubner \& Abate's and Durbin's inverse transform techniques. Results are presented in Figure 6 for the same ratio $\left(\eta_{1} / E_{1}=\eta_{2} / E_{2}=0.1\right)$ as in Example 1 . Fluctuation is observed in the time-dependent displacement and bending moment values at the center of the plate under the load Type II as the time increases in the MDOP inverse transform technique whereas there is no fluctuation in the Dubner \& Abate's and Durbin's inverse transform methods.

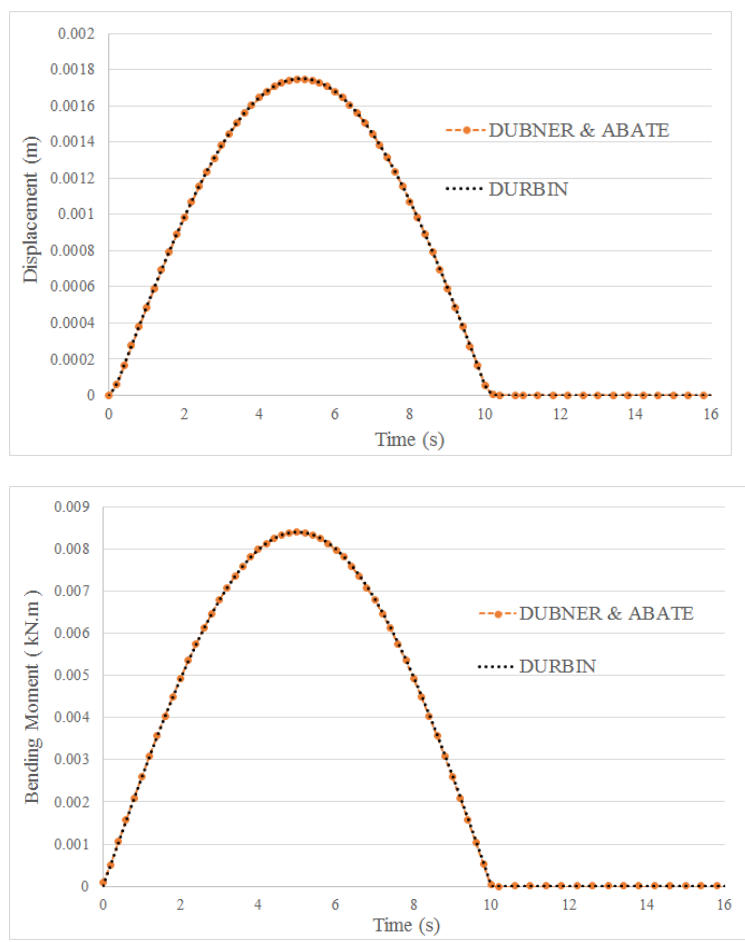

Figure 6. Displacement and Bending Moment-time variation

\section{Example 3}

In this example, a simply supported viscoelastic plate subjected to the triangular impulsive load (Type III) for $\mathrm{t}_{1}=10 \mathrm{~s}$ is considered for different $\eta / E$ ratios in order to show the damping effect in the central displacement-time variation. For the numerical inversion MDOP, Dubner \& Abate's and Durbin's transform techniques are employed. However, only the results of the Durbin's transform technique is presented in Figure 7. As expected, viscoelastic behaviour of the plate approaches the elastic behaviour when decreasing the viscosity coefficient $(\eta)$.

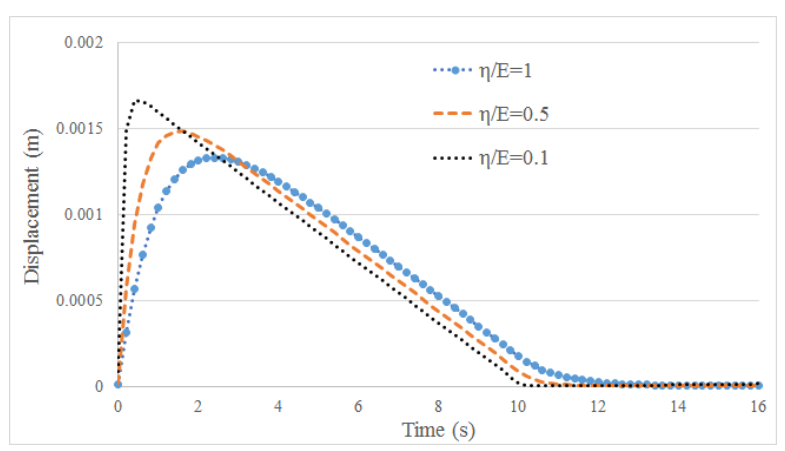

Figure 7. Effect of viscosity coefficient on the amplitude of displacement. 


\section{Conclusion}

In this study, the mixed finite element method in the Laplace-Carson space is developed for the quasi-static analysis of viscoelastic Kirchhoff plates with the model of four-parameter solid type. The functional in the Laplace-Carson space, which is derived through a systematic procedure based on the Gâteaux Differential, has four independent variables in addition to the geometric and dynamic boundary conditions. For transformation of the numerical results in the LaplaceCarson domain to the real time domain, various inverse transform techniques such as Maximum Degree of Precision (MDOP), Dubner and Abate's, and Durbin's methods are employed. The performance of the inverse transform methods and the presented mixed finite element formulation is tested through various quasi-static problems.

\section{Acknowledgements}

This research is supported by the Scientific and Technological Research Council of Turkey under the grant number 213M332. The authors gratefully acknowledge this support.

\section{References}

1. W. Flügge, Viscoelasticity, second ed., Springer: Berlin (1975)

2. R.M. Christensen, Theory of Viscoelasticity, second ed., Academic Press: New York (1982)

3. J.L. White, Finite elements in linear viscoelastic analysis. Proc. of the 2nd Conference on Matrix Method in Structural Mechanics. AFFDL-TR-68-150: 489-516 (1986)

4. T. Chen, The hybrid Laplace transform / finite element method applied to the quasi-static and dynamic analysis of viscoelastic Timoshenko beams. Int. J. Numer. Meth. Eng. 38,509-522 (1995)

5. Y.Z. Wang, T.J. Tsai, Static and dynamic analysis of a viscoelastic plate by the finite element method. Appl. Acoust. 25, 77-94 (1988)

6. S. Yi, H.H. Hilton, Dynamic finite element analysis of viscoelastic composite plates. Int. J. Numer. Meth. Eng. 37, 4081-96 (1994)

7. M.H. Ilyasov, A.Y. Aköz, The vibration and dynamic stability of viscoelastic plates. International Journal of Engineering Sciences. 38,695-714 (2000)
8. B. Temel, M.F. Şahan, Transient analysis of orthotropic, viscoelastic thick plates in the Laplace domain. European Journal of Mechanics A/Solids. 37, 96-105 (2013)

9. A.Y. Aköz, F. Kadığlu, G. Tekin, Quasi-static and dynamic analysis of viscoelastic plates. Mechanics of Time-Dependent Materials, DOI 10.1007/s11043015-9274-8 (2015)

10. A.Y. Aköz, F. Kadıoğlu, The mixed finite element method for the quasi-static and dynamic analysis of viscoelastic Timoshenko beams. Int. J. Numer. Meth. Eng. 44, 1909-32(1999)

11. F. Kadıoğlu, A.Y. Aköz, The mixed finite element method for the dynamic analysis of visco-elastic circular beams. Proc. of the 4th International Conference on Vibration Problems, Jadavpur University (1999)

12. F. Kadıŏlu, A.Y. Aköz, The quasi-static and dynamic responses of viscoelastic parabolic beams. Proc. on the 11th National Applied Mechanics Meeting (in Turkish). Bolu-Turkey (2000)

13. F. Kadığlu, A.Y. Aköz, The mixed finite element for the quasi-static and dynamic analysis of viscoelastic circular beams. International Journal of Structural Engineering and Mechanics. 15,735-752 (2003)

14. S.P. Timoshenko, S. Woinowsky-Krieger, Theory of Plates and Shells. McGraw-Hill Book Company (1959)

15. C.L. Dym, I.H. Shames, Solid Mechanics: A Variational Approach. McGraw-Hill: New York (1973)

16. J.T. Oden, J.N. Reddy, Variational Methods in Theoretical Mechanics. Springer-Berlin (1976)

17. H. Dubner, J. Abate, Numerical inversion of Laplace transforms by relating them to the finite Fourier cosine transform. J. ACM. 15,115-123(1968)

18. VI, Krylov, N.S. Skoblya, Handbook of numerical inversion of Laplace transforms. Translated from Russian, Israel Program for Scientific Translations, Jerusalem (1969)

19. F. Durbin, Numerical inversion of Laplace transforms: An efficient improvement to Dubner and Abate's method. Comput. J. 17,371-376 (1974)

20. G.V. Narayanan, D.E. Beskos, Numerical operational methods for time-dependent linear problems. Int. J. Numer. Meth. Eng. 18, 1829-1854 (1982) 